\title{
Davide Monda, Il potere mirabile di un grande amore. Idee e sentimenti nella civiltà letteraria europea
}

\section{Gabriella Bosco}

\section{(2) OpenEdition}

1 Journals

\section{Edizione digitale}

URL: http://journals.openedition.org/studifrancesi/7779

DOI: $10.4000 /$ studifrancesi. 7779

ISSN: 2421-5856

\section{Editore}

Rosenberg \& Sellier

\section{Edizione cartacea}

Data di pubblicazione: 1 décembre 2009

Paginazione: 687-688

ISSN: 0039-2944

\section{Notizia bibliografica digitale}

Gabriella Bosco, "Davide Monda, Il potere mirabile di un grande amore. Idee e sentimenti nella civiltà

letteraria europea», Studi Francesi [Online], 159 (LIII | III) | 2009, online dal 30 novembre 2015, consultato il 09 janvier 2021. URL: http://journals.openedition.org/studifrancesi/7779 ; DOI: https://doi.org/ 10.4000/studifrancesi.7779

Questo documento è stato generato automaticamente il 9 janvier 2021.

\section{(c) (1)}

Studi Francesi è distribuita con Licenza Creative Commons Attribuzione - Non commerciale - Non opere derivate 4.0 Internazionale. 


\title{
Davide Monda, Il potere mirabile di un grande amore. Idee e sentimenti nella civiltà letteraria europea
}

\author{
Gabriella Bosco
}

\section{NOTIZIA}

DAVIDE MONDA, Il potere mirabile di un grande amore. Idee e sentimenti nella civiltà letteraria europea, premessa di Matteo VERONESI, postfazioni di Edoardo RIPARI e Lorenzo TINTI, Napoli, Liguori Editore, 2009, pp.263.

1 Prosecuzione del precedente Amore e altri despoti (2004), il volume raccoglie una serie di saggi che affrontano diverse forme del sentimento amoroso, spaziando dalla civiltà letteraria della Roma imperiale a quella dell'Europa primonovecentesca. Davide MONDA fa opportuno ricorso, per il suo discorso critico che questa volta include, accanto all'amore, la tematica della marginalità sociale, alla scrittura in prima persona, facendoci entrare nelle sue letture attraverso il prisma dell'esperienza. I tredici capitoli studiano tanto l'ardente e travagliato amore di Sant'Agostino per Dio quanto quello di Erasmo da Rotterdam per una pace giusta, tanto l'eros neoplatonico-cristiano di Gravina quanto la passione per il viaggio in età moderna, il sentimento tragico che anima il Werther goethiano come la compassione autentica per gli sventurati, e molte altre varianti ancora. Né Monda si esime dall'offrirci, in ossequio all'amore per le bonae litterae, le sue note "per una didattica efficace delle idee e delle lettere in età postmoderna». Ogni saggio è corredato da dense pagine di riferimenti bibliografici assolutamente preziosi.

2 Matteo Veronesi firma una premessa intitolata Dalla cognitio rerum alla cognitio signorum. Sul «positivismo trascendentale» di Davide Monda (pp. VII-X), che fa riferimento all'espressione usata da Ezio Raimondi per pensatori come Dilthey o Simmel, «sospesi scrive Veronesi, ritraendo Monda per interposta persona - fra l'eredità del 
fenomenismo, dell'accertamento, della concretezza tardo-ottocenteschi e le inquietudini e le aperture, già imminenti, del Novecento neo-idealista e fenomenologico, che non ignora né opacizza l'evento, il dato, il "fatto", pur immergendoli nella controluce trepida ed inverante di un cielo ulteriore».

3 Specularmente, a chiusura del volume, due testi lo mettono in prospettiva. Il primo, di Edoardo Ripari (Il potere mirabile della storia delle idee, pp. 254-256), analizza il metodo di lavoro dell'autore e sottolinea «l'alto profilo etico-civile del suo diuturno, instancabile colloquio con taluni grandi autori del passato». Il secondo, di Lorenzo Tinti, è invece rivolto direttamente a Davide Monda (Notula morale foggiata a mo' di lettera, ove si rimeditano le pagine del libro come dialogando con l'autore, pp. 257-263), e anch'esso come quello introduttivo di Veronesi rievoca il maestro comune Raimondi, per ripercorrere poi i capitoli del libro alla luce del suo insegnamento, e concludersi con una considerazione sul futuro della cultura, ispirata dall'ultimo saggio del volume, tratta da un testo di Massimo Cacciari del 2002, Brevi inattuali sullo studio dei classici: «Temo non esista peggior utopia regressiva di quella che anima oggi gli apologeti della scuolainformazione, della scuola-azienda, della scuola produzione di impiegati (...) Chi abbia letto una sola tragedia greca, una sola invettiva dantesca, un verso della Ginestra, saprà ascoltare, saprà riconoscere i propri limiti e il valore altrui - ma passivamente obbedire mai». 